\title{
Hou Gu Mi Xi
}

National Cancer Institute

\section{Source}

National Cancer Institute. Hou Gu MiXi. NCI Thesaurus. Code C143159.

An orally bioavailable dietary supplement and a formulation derived from the traditional

Chinese medicine (TCM) Shen Ling Bai Zhu San, that can potentially be used to ameliorate spleen deficiency and improve symptoms of digestive disorders. Shen Ling Bai Zhu San is composed of ginseng, tuckahoe, atractylodes, baked licorice, coixenolide, Chinese yam, lotus seed, shrinkage fructus amomi, platycodon grandiflorum, white hyacinth bean, and dried orange peel. In Hou Gu Mi Xi, atractylodes and platycodon grandiflorum are removed from Shen Ling Bai Zhu San and perilla leaf is added. Upon oral administration, Hou Gu Mi Xi replenishes qi, and may improve spleen qi deficiency, gastrointestinal (Gl) symptoms and Gl function. 